\title{
Surface-induced dissociation of anionic vs cationic native-like protein complexes
}

Sophie R. Harvey, Zachary L. VanAernum, Vicki H. Wysocki

Department of Chemistry and Biochemistry and Resource for Native Mass Spectrometry Guided Structural Biology, The Ohio State University, Columbus, Ohio, 43210, United States

\section{Abstract}

Characterizing protein-protein interactions, stoichiometries, and subunit connectivity is key to understanding how subunits assemble into biologically-relevant, multi-subunit protein complexes. Native mass spectrometry (nMS) has emerged as a powerful tool to study protein complexes due to its low sample consumption and tolerance for heterogeneity. For such nMS studies, positive mode ionization is routinely used and charge reduction, through the addition of solution additives, is often used, as the resulting lower charge states are often more compact and considered more native-like. When fragmented by surface-induced dissociation, charge reduced complexes often give increased structural information over their "normal-charged" counter parts. A disadvantage of charge-reduction is that increased adduction, and hence peak broadening, is often observed when charge-reducing solution additives are present. Recent studies have shown that protein complexes ionized using negative mode generally form in lower charge states relative to positive mode. Here we demonstrate that the lower charged protein complex anions, activated by SID in an ultrahigh mass range Orbitrap mass spectrometer, fragment in a manner consistent with their solved structures, hence providing substructural information. Negative mode ionization in ammonium acetate offers the advantage of charge reduction without the peak broadening associated with solution phase charge reduction additives and provides direct structural information, when coupled with SID. SID of 20 S human proteasome (a 28-mer comprised of four stacked heptamer rings in an $\alpha \beta \beta \alpha$ formation), for example, provides information on both substructure (e.g., splitting into a $7 \alpha$ ring and the corresponding $\beta \beta \alpha 21$-mer; and into $\alpha$ dimers and trimers to provide connectivity around the 7a ring) and proteoform information on monomers. 


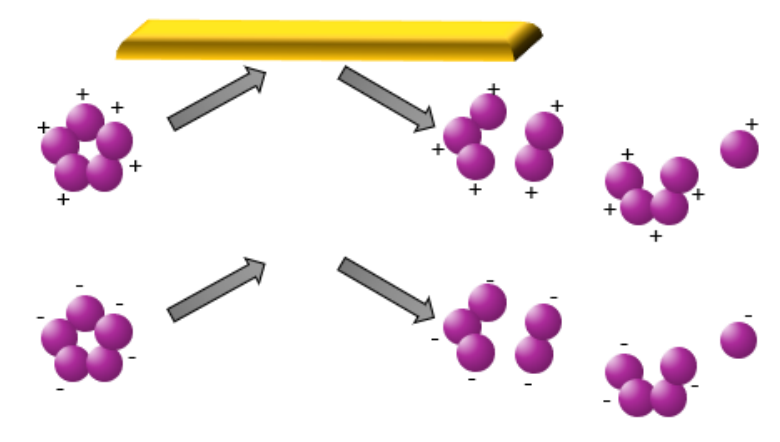

\section{Introduction}

Within the cell, a host of biological processes are mediated through multicomponent protein complexes. ${ }^{1}$ Characterization of these protein complexes, including the stoichiometry, topology, identification of all binding partners, and classification of posttranslational modifications is essential to better understand how these complex systems carry out their function and how misfolding or alterations in architecture can lead to malfunction. ${ }^{2,3}$ While classical techniques, such as X-ray crystallography, NMR, and, more recently, cryo-EM, can provide high-resolution structures these techniques have limitations, often high concentrations and high-purities are required and conformationally dynamic systems can still pose challenges ${ }^{4-6}$ Since the advent of electrospray ionization, mass spectrometry has emerged as a powerful tool to study large proteins and protein complexes. ${ }^{7-10}$ Using appropriate electrolyte solutions, typically ammonium acetate $(A m A c)$, and gentle desolvation and instrumental conditions, intact complexes, including protein:protein and protein:ligand complexes, can be transferred into the gas-phase, kinetically trapped in native-like structures. ${ }^{11-13}$ Once transferred into the gas-phase, information on their stoichiometry, substructure, and conformations can be obtained using dissociation techniques or ion mobility measurements. ${ }^{14-16}$ While being a lower resolution than traditional structural characterization methods, mass spectrometry offers advantages in low sample consumption (typically $\mu \mathrm{L}$ of sample at $\mu \mathrm{M}$ concentrations) and can be used to study dynamic and heterogenous systems. ${ }^{17}$ 
Most protein MS studies have been conducted on protein cations. Several recent studies have, however, utilized negative ionization mode. ${ }^{18-21}$ Interestingly, these studies reported that the protein anions have lower average charge states than the corresponding cations which has resulted in discussions regarding the process by which these molecules are ionized. ${ }^{19,20}$ Several electrospray mechanisms have been previously proposed, with discussion focusing on positive ionization mode, and are thought to depend on the nature of the molecule. ${ }^{22-24}$ In positive mode electrospray, it has been proposed that globular proteins follow the charged residue model, producing ions with a composition close to $\left[\mathrm{M}+\mathrm{z}_{\mathrm{R}} \mathrm{H}\right]^{2 \mathrm{R}+}$, where $\mathrm{z}_{\mathrm{R}}$ is the Rayleigh charge of protein-sized water droplets. Previous studies have shown that for proteins and protein complexes, positive charge states observed are approximately $90 \%$ of the Rayleigh model predicted charge, however, observed negative charge states are only approximately $70 \%$ of the predicted charge. ${ }^{19}$ This difference has been attributed to the emission of small charge-carrier ions in the final stages of desolvation. In the case of AmAc solutions, the larger deprotonated acetate anion is more volatile than the protonated ammonium cation, therefore fewer charges remain in the negatively charged ion droplets compared to positively charged ion droplets, and hence resulting in the lower charged protein anions with less adduction. ${ }^{19,24,25}$ Interestingly, despite their lower charge states, ion mobility has shown that cations and anions have similar collisional cross sections (CCS). ${ }^{20}$ The similar CCS suggest that similar conformational families might be sampled in each case and hence negative mode ESI could be beneficial in studies when lower charge states are desired.

Many native mass spectrometry (nMS) studies are conducted using "charge-reducing" reagents as lower charge states can give more stable, native-like structures and fragmentation products. ${ }^{26-}$ 28 Charge reduction is typically achieved using solution-phase additives such as triethylammonium acetate (TEAA), which is an efficient charge reduction reagent; however, TEAA can lead to peak broadening due to increased adduction on the ion. ${ }^{29}$ Here we seek to determine 
if the lower charge state protein complex anions generated through negative ESI can be used as an alternative to solution-phase charge reduction in nMS protein structural studies, offering the advantage of lower charge states without the increased adduction.

nMS studies commonly employ gas-phase dissociation methods to obtain substructural information. The most commonly used method of dissociation is collision-induced dissociation (CID).$^{30}$ In CID, ions are accelerated into a neutral buffer gas, and the analytes undergo multiple collisions with the very low mass collision gas. For protein complexes, the large number of low energy collisions means that CID typically proceeds via an unfolding or restructuring mechanism producing unfolded, highly charged monomer and the complementary n-1 mer. ${ }^{31-33}$ Hence CID provides limited direct information on substructure and assembly ( $n$-mers of different structure/connectivity produce monomer and $\mathrm{n}-1$ mer). An alternative method of dissociation used in nMS studies is surface-induced dissociation (SID). In SID, analytes are accelerated towards, and collide against, a surface, a collision target that is more massive than the complex. This energy deposition process results in a sudden energy jump and dissociation typically occurs with less unfolding than CID and in a manner that is consistent with the solved structure. ${ }^{14,34-36}$ Note, however, that products of the same charge state produced by CID or SID tend to have the same CCS values but SID tends to produce lower charged products and CID higher charged products. ${ }^{37}$ Recently, it was shown that the SID spectra produced from complexes analyzed under gentle instrumental conditions vs. intentional pre-activation are different, and hence SID can be used to assess structure and structural rearrangement. ${ }^{38}$ Here we use negative mode nano-ESI (nESI) and SID to study the substructure of several model protein complexes, highlighting the utility of this method for structural characterization. Comparisons with positive mode spectra are also provided. 


\section{Methods}

\section{Materials}

C reactive protein was purchased from Sigma Aldrich. TRAP was expressed in Escherichia coli BL21(DE3) cells, purified and refolded as previously described. ${ }^{39,40}$ Samples were buffer exchanged into $200 \mathrm{mM}$ AmAc or $400 \mathrm{mM}$ AmAc, respectively using Biorad micro P6 spin columns. Human proteasome was purified by FLAG affinity purification from HEK293 cells as previously described and free FLAG peptide was present in the analyzed samples. ${ }^{41}$ Human proteasome was received from the lab of Michal Sharon in $500 \mathrm{mM} \mathrm{AmAc}$ and diluted to $150 \mathrm{mM}$ AmAc before analysis. Samples were run at approximately $700 \mathrm{nM}-1 \mu \mathrm{M}$ protein concentration. AmAc concentrations for individual samples were based on previously optimized conditions. ${ }^{40,42,43}$

\section{Instrumentation}

All experiments were conducted on a Thermo Q Exactive Ultra High Mass Range (UHMR) Orbitrap MS (Bremen, Germany), which has been modified to include SID, as described previously. ${ }^{44,45}$ In brief, a 4cm SID device is inserted in place of the transfer multipole between the selection quadrupole and the C-trap. Voltages were supplied to the SID cell via external dc power supplies (Ardara Technologies, Ardara, PA) and controlled through the accompanying Tempus Tune software (Ardara Technologies, Ardara, PA). As described previously, ${ }^{44}$ in order to increase the SID voltage, and hence energy range, the C-trap offset voltage is also supplied with an external power supply. In positive mode this involves setting the $C$ trap offset voltage low relative to up-stream optics and in negative mode, setting the $C$ trap offset voltage high relative to upstream optics. The surface was prepared as follows; a $17-\mathrm{mm} \times 13-\mathrm{mm} \times 0.5-\mathrm{mm}$ gold surface slide, $1,000 \AA$ of Au on $50 \AA$ of Ti on glass (EMF Corp.), was cleaned for 15 min in a UV cleaner (Model 135500; Boekel Scientific). The cleaned surface was then incubated in $\sim 3 \mathrm{~mL}$ of $1 \mathrm{mM}$ solution of alkanethiol perfluorinated on the final 10 carbons of the 12 carbon chain $\left(\mathrm{FC}_{12}\right)$ in 
ethanol in the dark. $\mathrm{FC}_{12}$ was synthesized using a previously reported protocol. ${ }^{36}$ Following incubation, the surface was cleaned by sonicating in $\sim 3 \mathrm{~mL}$ of ethanol for $1 \mathrm{~min}$; this cleaning process was repeated six times with fresh aliquots of ethanol.

All samples were ionized via static nanospray ionization using in-house pulled borosilicate capillaries with the electrospray voltage applied to a platinum wire in contact with the solution. A capillary temperature of $200^{\circ} \mathrm{C}$ was applied and a trap gas flow rate of 5-9 was used, depending on the size of the system studied with higher gas flows used for larger systems. The resolution, as defined at $m / z 400$, was set to 3,125 for all complexes studied here. In negative mode, we found that obtaining sufficient signal required higher voltages to be applied across the source optics. Positive mode data were acquired using the same source settings as negative to enable comparisons to be made between polarities using the same instrumental settings. Typical settings used here for transmission mode and SID can be found in the supporting information (Table S1). SID is reported here for single charge state studies as SID energy (voltage ${ }^{*}$ charge state). For comparisons of different charge states of the same protein, different SID voltages were chosen in order for similar to energies to be compared.

\section{Results and discussion}

We first considered homopentameric C-reactive protein (CRP), a model protein system which has been previously studied with SID. ${ }^{37,38,43}$ We studied CRP ionized in positive mode and prepared under both "normal" charge conditions (200 mM ammonium acetate (AmAc)), and "chargereduced" conditions (160 mM AmAc +40 mM triethylammonium acetate (TEAA)). ${ }^{26,46}$ Chargereduced conditions result in a lowering of the average charge state from $+23.38 \pm 0.58$ to +17.47 \pm 0.16 (Figure S1), for SID studies we selected the most abundant charge state from each condition. The lower average charge states observed in TEAA are commonly exploited in structural studies, particularly using SID as increased structural information can be obtained from these lower charged, often more compact species, as discussed previously and demonstrated in 
Figure 1. ${ }^{36,37}$ Low energy SID (1152 eV) of the 24+ CRP pentamer in AmAc produces primarily monomer and dimer with some low intensity trimer also observed, Figure 1A and Figure S2A. However, the lower-charged CRP pentamer (18+) fragments to monomer+tetramer and dimer+trimer with low energy SID (1152 eV) as shown in Figure 1B and Figure S2B, consistent with the cyclic structure of this complex ${ }^{47}$ Hence, the addition of TEAA and dissociation of lowercharge states is advantageous in SID structural studies. However, the addition of TEAA can often result in increased adduction and hence increased peak width, ${ }^{29}$ which can be problematic in ligand binding studies when high mass accuracy is required. Given the previous reports of lower average charge states for proteins when analyzed in negative ionization mode, we next studied CRP prepared in $200 \mathrm{mM} A m A c$ but ionized in negative ionization mode. In negative mode $\mathrm{nESI}$ the average charge state of CRP is significantly lower than when analyzed in positive mode ($18.98 \pm 0.08$ vs $+23.38 \pm 0.58$, Figure $S 1)$. Interestingly, the average charge state is only slightly higher in negative mode than when analyzed under charge reducing conditions in positive mode $(-18.98 \pm 0.08 v s+17.47 \pm 0.16)$. To determine if the same level of structural information could be obtained from CRP anions as was obtained for charge-reduced cations, we subjected the most abundant charge state (19-) to low energy SID (1140 eV), Figure 1C and Figure S2C. SID of 19CRP produced monomer+tetramer and dimer+trimer, consistent with the solved pentameric ring structure, suggesting that the lower charge states obtained from negative mode ionization can be exploited in protein structural studies.

Previous reports have shown that SID can probe structural rearrangement in addition to native structures. ${ }^{38,48}$ One such study intentionally activated CRP using increasing voltages in the source region and then measured the unfolding/rearrangement with ion mobility and SID. ${ }^{38}$ To determine if negative mode SID can also be used to probe structural rearrangements, we performed a similar experiment in this case utilizing increasing in-source trapping voltages on the UHMR instrument. We considered CRP prepared under charge reducing conditions in positive mode and in AmAc in 
negative mode, both of which were able to provide structural information with SID with gentle insource trapping (Figure 1). In both cases, increasing the in-source trapping voltage from $\pm 30 \mathrm{~V}$ to $\pm 100 \mathrm{~V}$ appeared to have a little effect on the full MS (MS1), with pentamer remaining the dominant product and no shift in charge state distribution observed (Figure S3). When SID was performed on the dominant charge state (18+ or 19-) at low in-source trapping voltages we see fragments consistent with a cyclic pentamer, monomer+tetramer and dimer+trimer (Figure 2 and Figure S4).

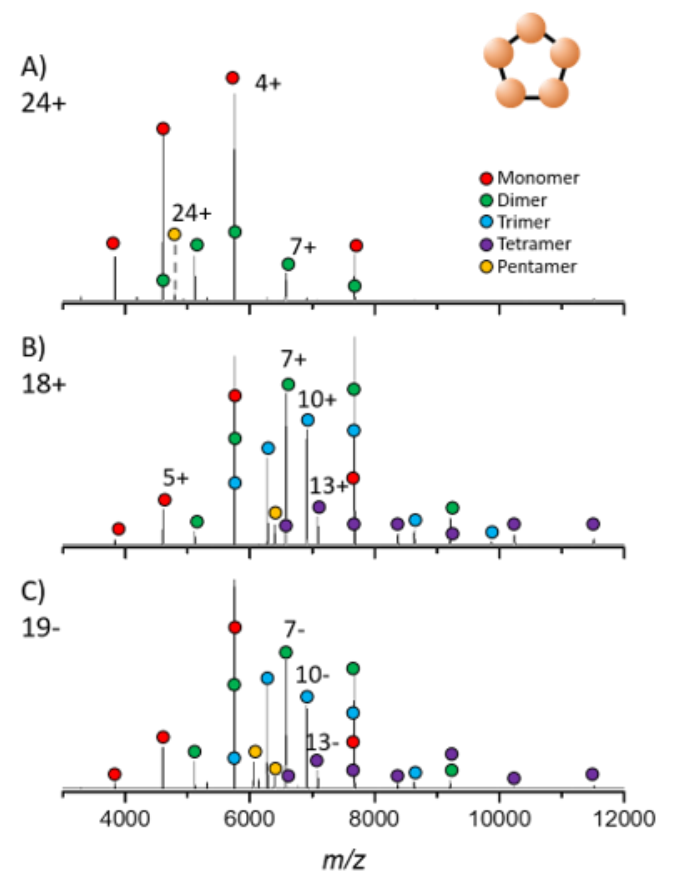

Figure 1: Low-energy SID of CRP, acquired with an in-source trapping voltage of $\pm 30 \mathrm{~V}$. A) $24+\mathrm{CRP}$ from $200 \mathrm{mM}$ AmAc acquired at an SID energy of $1152 \mathrm{eV}$ B) 18+ CRP from $160 \mathrm{mM} \mathrm{AmAc}+40 \mathrm{mM}$ TEAA, acquired at an SID energy of $1152 \mathrm{eV}$ and C) 19- CRP from $200 \mathrm{mM}$ AmAc, acquired at an SID energy of $1140 \mathrm{eV}$. Inset is cartoon representation of structure (PDB 1GNH). ${ }^{47}$ Deconvoluted data is shown in Figure S2.

However, as the in-source trapping voltage is increased we begin to see decreased dissociation by SID, with higher abundant precursor remaining, and a shift in the dissociation products produced in both positive and negative mode. At high in-source trapping voltage, the dimer+trimer 
products decrease in intensity and the majority of pentamer dissociates to monomer+tetramer by SID. This shift in the observed SID dissociation pathway, as in-source trapping voltage is increased, is consistent with the protein restructuring/unfolding during in-source trapping activation and SID measuring the altered structure. It should be noted that different charge states are studied in negative vs positive mode $(-19$ vs +18$)$, this could, in-part, explain why the shift in dissociation patterns occurs at lower in-source trapping voltages in negative mode compared with positive mode. It is also possible that the different location of charges on the anions vs cations could result in gas-phase structural differences and different patterns of restructuring during insource trapping. In future work, collision-induced unfolding may help characterize these differences. The fact that the expected fragmentation pattern without source activation and altered fragmentation pattern after in-source trapping are observed in both positive mode and negative mode suggests that under gentle desolvation and instrumental conditions native protein structures can be preserved and studied in nMS using either polarity and that, like positive mode, negative mode SID can be used to probe structural changes.

We next considered Trp RNA binding attenuation protein (TRAP), which is a $91 \mathrm{kDa}$ cyclic homo 11-mer. Like CRP, TRAP presents with a lower average charge state in negative mode in 400 mM AmAc compared to positive mode $(-16.12 \pm 0.08 v s+21.08 \pm 0.25)$ and with a slightly higher average charge in negative mode than when prepared in charge reducing conditions, $320 \mathrm{mM}$ AmAc +80 mM TEAA, $(+15.27 \pm 0.10)$, as shown in Figure S5. Under each of the three conditions studied here we selected the most intense charge state for SID studies, 21+, 15+ and 16-. TRAP is a homomeric cyclic 11-mer, with equal interfaces between subunits, and therefore by SID we would expect it to dissociate to all possible subcomplexes (monomer to decamer). When TRAP is prepared in AmAc and subjected to low energy SID, multiple dissociation products are observed from monomer to hexamer but the larger multimers expected for this complex are not observed (Figure 3A and Figure S6A), consistent with previous studies for non-charge reduced samples; 
this may reflect secondary dissociation of the initial higher order oligomeric product ions. ${ }^{37}$ Under charge reducing conditions in positive mode, all expected oligomers can be observed from monomer to undecamer, consistent with the cyclic structure of this complex (Figure 3B and Figure S6B). When the lower charge state TRAP anions are studied by SID, like CRP, structural information can also be obtained with all expected subcomplexes observed. (Figure $3 \mathrm{C}$ and Figure S6C). A shift to the lower oligomeric states is observed for the 16- TRAP compared to the 15+ TRAP which could be due to the higher charge state species studied in negative mode. This once again highlights that it is possible to utilize the lower charge state anions for structural studies using SID, without having to add any solution additives. 


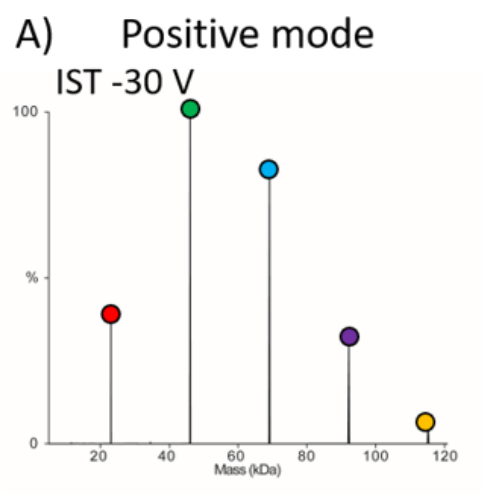

B) Negative mode
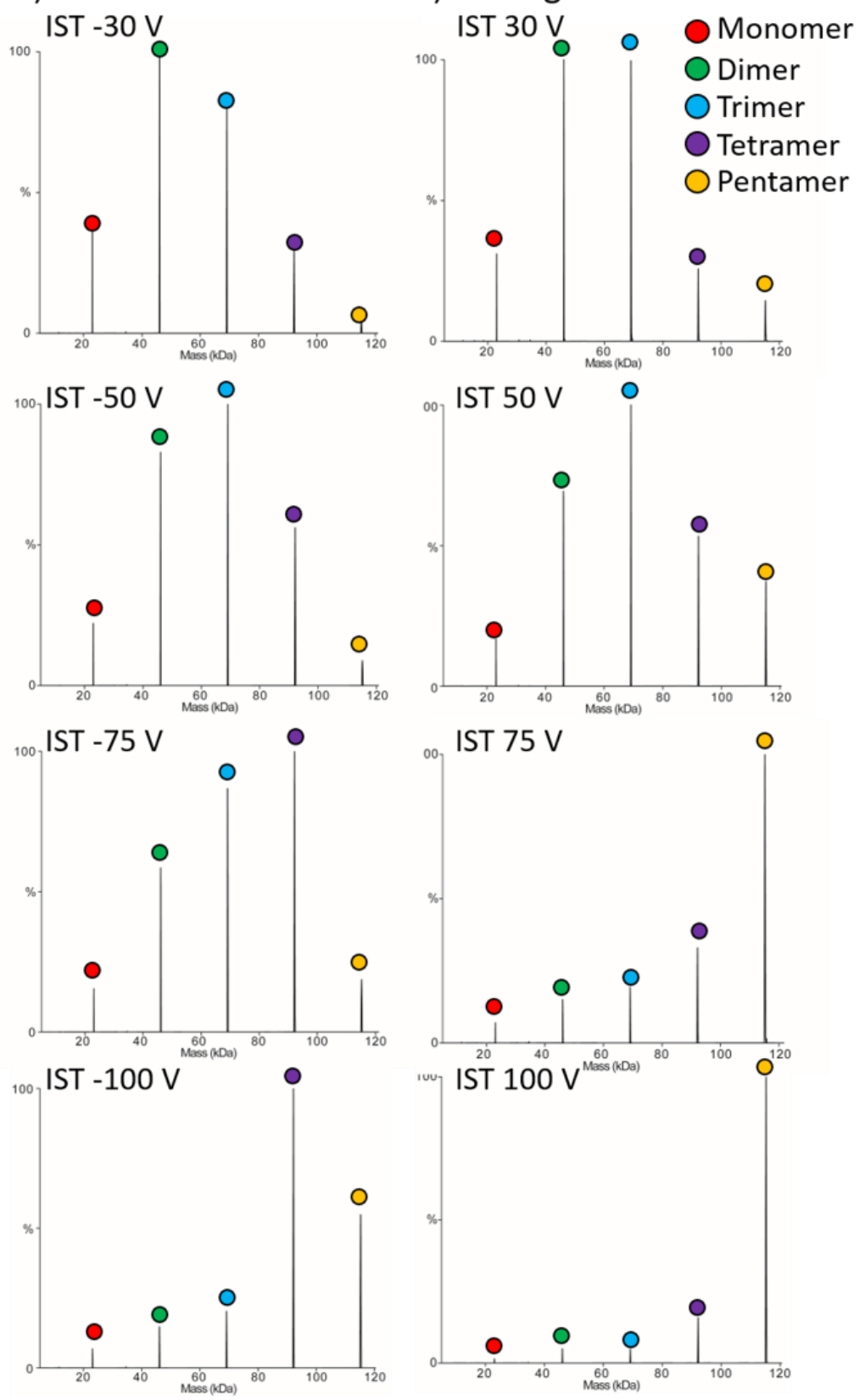

Figure 2: Deconvolved (mass-only domain) low-energy SID of CRP, acquired with increasing in-source trapping voltages A) 18+ CRP from $160 \mathrm{mM} \mathrm{AmAc}+40 \mathrm{mM}$ TEAA, acquired at an SID energy of $1152 \mathrm{eV}$ and B) 19- CRP from $200 \mathrm{mM}$ AmAc, acquired at an SID energy of $1140 \mathrm{eV}$. Data deconvolved using UniDec ${ }^{49}$, raw data can be found in Figure S4 

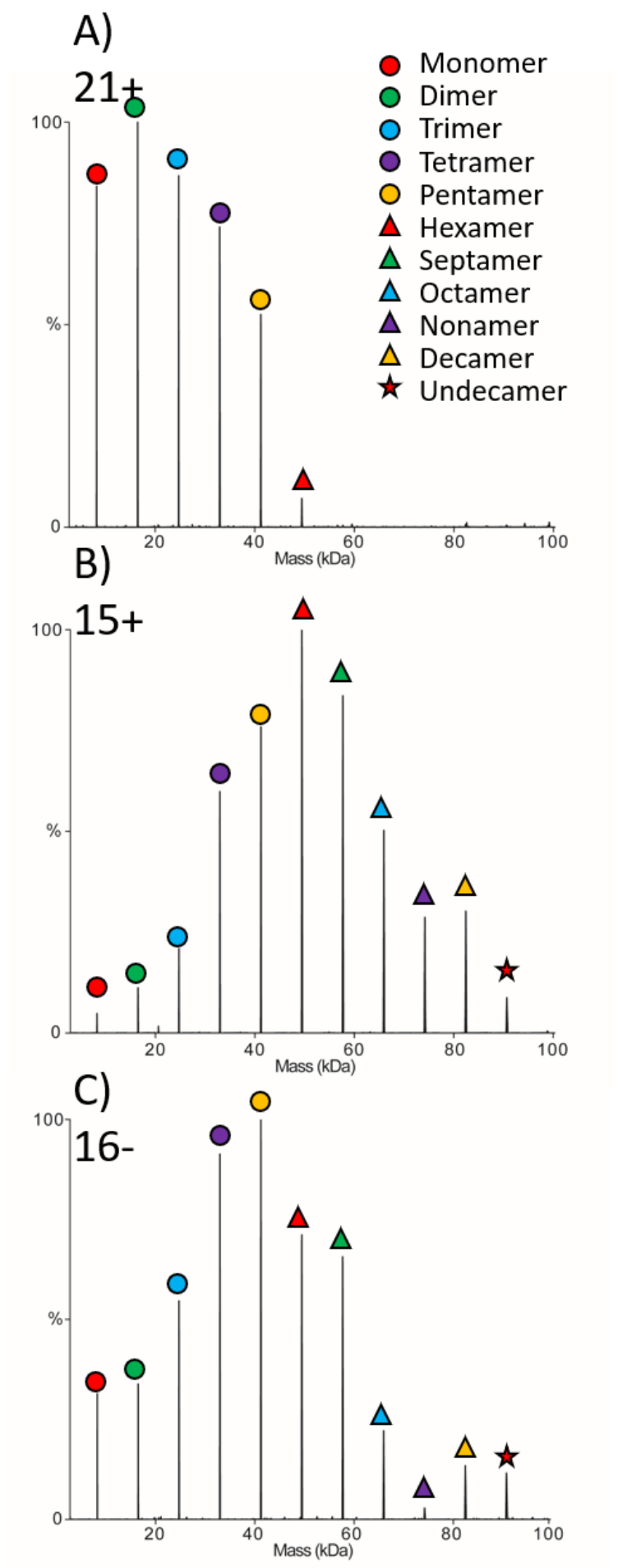

Figure 3: Low-energy SID of TRAP, acquired with an in-source trapping voltage of $\pm 5 \vee$ A) $21+$ TRAP from $400 \mathrm{mM}$ AmAc acquired at an SID energy of $1050 \mathrm{eV}$ B) 15+ TRAP from $320 \mathrm{mM} \mathrm{AmAc}+80 \mathrm{mM}$ TEAA, acquired at an SID energy of $1050 \mathrm{eV}$ and C) 16- TRAP from $200 \mathrm{mM} \mathrm{AmAc}$, acquired at an SID energy of $1056 \mathrm{eV}$. Data deconvolved using UniDec ${ }^{49}$, raw data can be found in Figure S6. 
Finally, we wanted to see if this approach could be extended to larger, hetero-oligomers so we also considered human 20 s proteasome. The 20 s proteasome is composed of 28 subunits ( $14 \alpha-$ type subunits and $14 \beta$-type subunits), which are arranged in a cylindrical structure consisting of four heptameric rings: the two outer rings are comprised of $\alpha$-type subunits and the two central rings are comprised of $\beta$-type subunits $\left(\alpha_{7} \beta_{7} \beta_{7} \alpha_{7}\right) .{ }^{50}$ As expected based on the results above, the average charge state decreases for proteasome when analyzed in $150 \mathrm{mM} \mathrm{AmAc}$ in negative mode compared to positive mode (centered around 49- vs 60+), and is slightly higher than when analyzed in 120 mM AmAc + 30 mM TEAA (centered around 49- vs 44+), Figure S7. In addition, under the soft instrumental conditions employed here, the average measured mass is similar when prepared in AmAc and analyzed in positive or negative mode (724, $056 \pm 301$ Da vs 724, $317 \pm 267 \mathrm{Da})$ and slightly larger than the theoretical mass of $716,224 \mathrm{Da}$ due to adducting and binding of the FLAG peptide (1012.97 Da) present in solution from purification. An increase in the measured mass is observed when the sample is prepared in AmAc+TEAA and analyzed in positive mode $(727,788 \pm 382 \mathrm{Da})$, demonstrating one of the challenges of using charge reducing reagents. For human 20s proteasome a single charge state was not selected for SID studies, instead a wide isolation window was applied around the whole charge state distribution, meaning the SID voltage cannot be converted to a single energy. Under all three conditions studied here (AmAc positive mode, AmAc+TEAA positive mode, and AmAc negative mode) at low voltage SID both the $\alpha_{7}$ ring and $\alpha_{7}+\beta_{7}$ half proteasome are observed, consistent with the structure and previous SID studies (Figure 4, and Table S2). ${ }^{32}$ PISA interfacial analysis reveals that the $\alpha . \beta$ interface is smaller, and therefore can be considered weaker than the $\beta-\beta$ interface, therefore, we would expect low energy SID to cleave the $\alpha_{-} \beta$ interface producing a free $\alpha_{7}$ ring and, if enough energy is input, to also cleave the $\beta-\beta$ interface, consistent with products observed. ${ }^{42,50,51}$ In addition, some free a monomer and dimers are observed when analyzed in positive mode from AmAc (Figure 4A). Increasing the SID voltage allows higher intensity a monomer, dimers, and trimers to be observed, therefore information on the proteoforms present for a monomers can be 


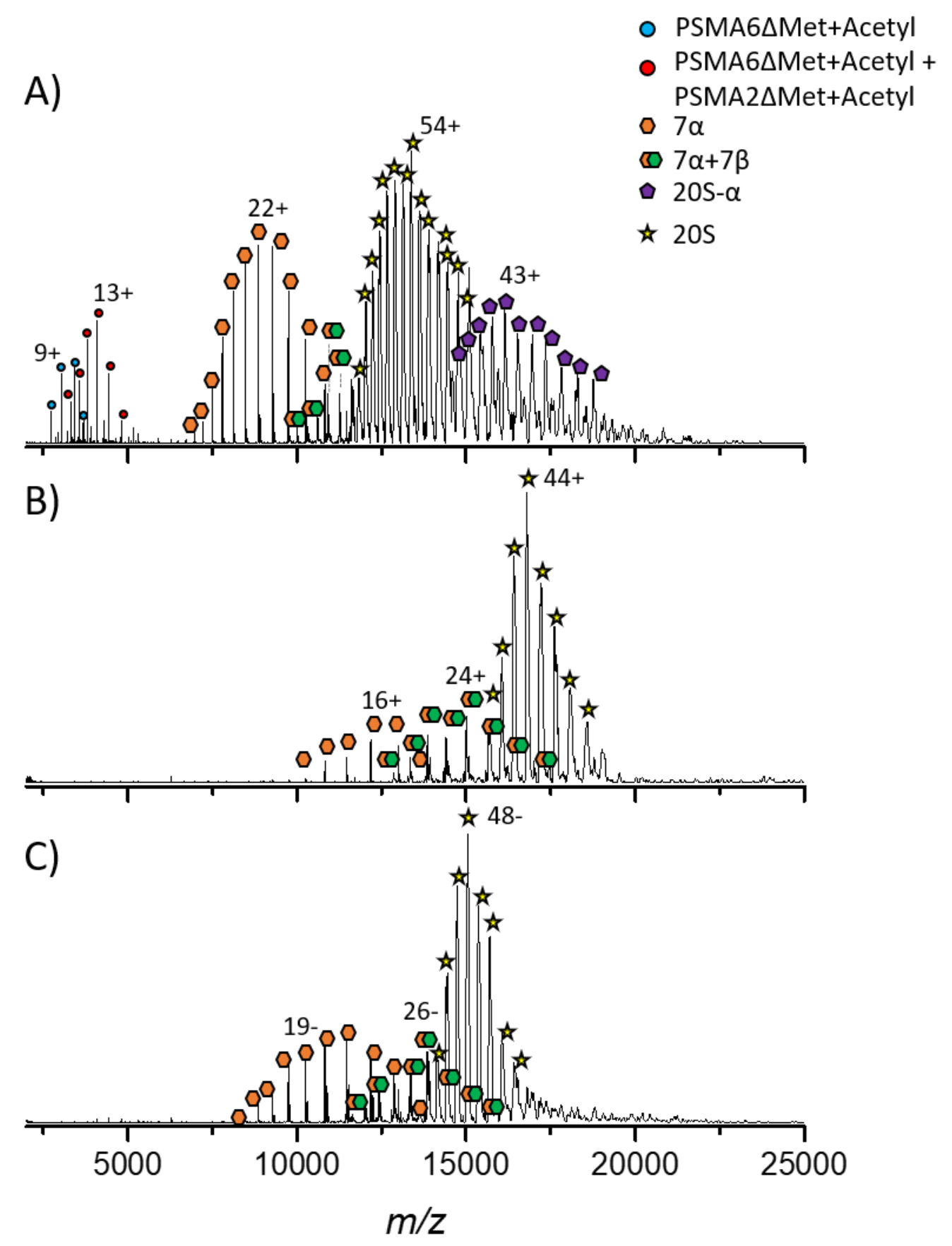

Figure 4: Low-energy SID of human proteasome, acquired with an in-source trapping voltage of $\pm 50 \mathrm{~V}$ A) proteasome in $150 \mathrm{mM}$ AmAc acquired at an SID voltage of $90 \mathrm{~V}$ in positive mode B) proteasome in 120 $\mathrm{mM} \mathrm{AmAc}+30 \mathrm{mM}$ TEAA acquired at an SID voltage of $130 \mathrm{~V}$ in positive mode and C) proteasome in 150 $\mathrm{mM} A m A c$ acquired at an SID voltage of $110 \mathrm{~V}$ in negative mode. Different SID voltages are given here for each condition to account for differences in the average charge state and hence SID energy under each condition. Note that experimental masses are higher in TEAA due to increased adducting on the precursor and are given in Table S2. 
obtained (Figure 5, Figure S8 and Table S3). In this case we find that AmAc in positive mode provides the most extensive coverage of proteoforms, as is expected given that under these conditions the highest charge states and most extensive dissociation of the 20s proteosome is observed. Therefore, for full a subunit proteoform characterization of the 20 s proteosome by SID, AmAc in positive mode would be preferred. However, further structural information can be obtained by considering the a dimers and trimers observed (Figure 5, and Table S4), as these can be used to confirm connectivity in the $7 \alpha$ ring ${ }^{42}$ For AmAc+TEAA insufficient coverage of the dimers and trimers does not allow the connectivity of the full $7 \alpha$ ring to be determined by SID. However, when prepared in AmAc and studied in either positive or negative mode, full connectivity of the $7 \alpha$ ring can be determined from the dimers and trimers offering an advantage over AmAc+TEAA studied in positive mode. This demonstrates that negative mode SID can be used to obtain substructural information on protein complexes of various sizes and complexity, and has the advantage of generating lower charge states without the need for additives that cause peak broadening and a corresponding decrease in spectral quality. Therefore, in the future, preparing samples in AmAc and analyzing by both positive and negative mode SID could provide increased structural and proteoform characterization. 


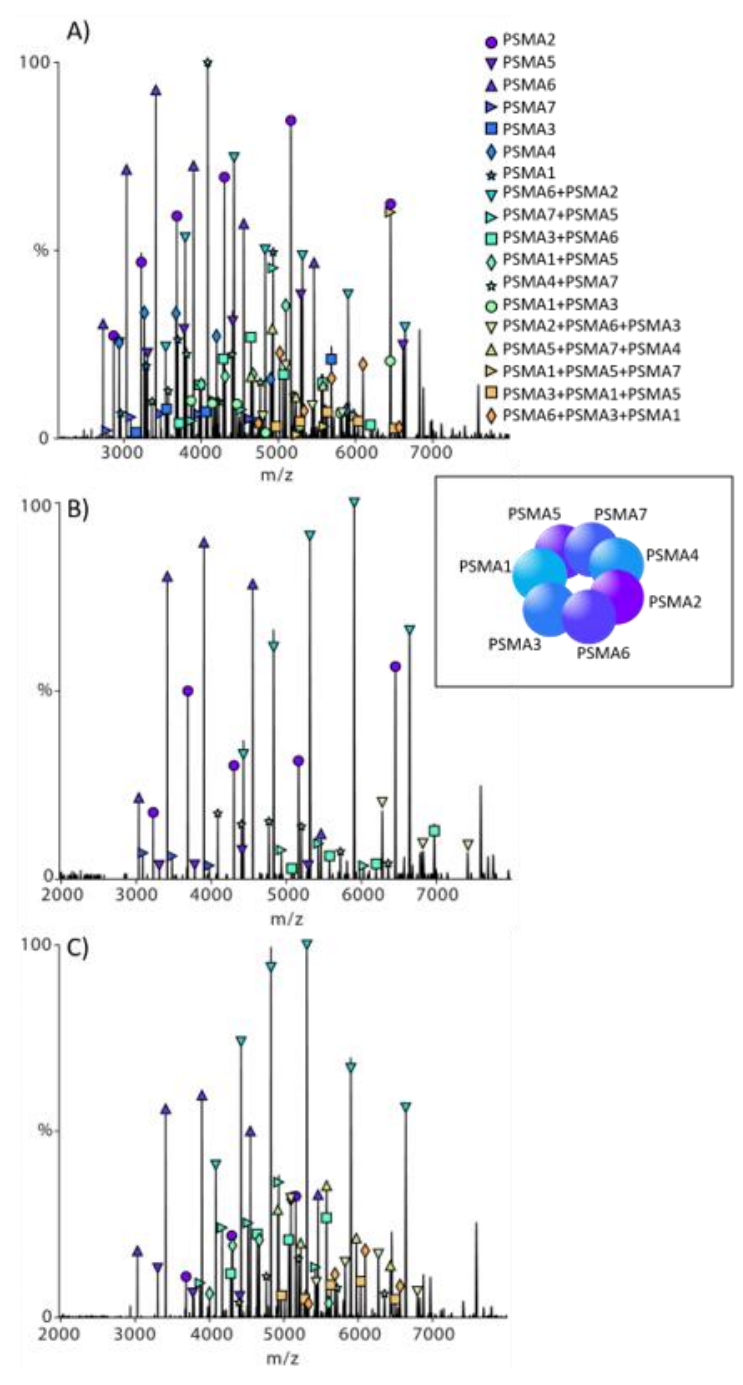

Figure 5: High energy SID of human 20s proteasome A) in $150 \mathrm{mM}$ AmAc in positive ionization mode, $190 \mathrm{~V} \mathrm{SID} \mathrm{B}$ ) in $120 \mathrm{mM} \mathrm{AmAc}+30 \mathrm{mM}$ TEAA in positive ionization mode, $210 \mathrm{~V}$ SID and C) in $150 \mathrm{mM}$ AmAc in negative ionization mode, $210 \mathrm{~V}$ SID. Shown here is zoom in of the low mass region, highlighting $\alpha$ - monomers and dimers, full MS range is shown in Figure S8. Inset is cartoon representation of $7 \alpha$ ring, determined in crystal structure. ${ }^{50}$ 


\section{Conclusions}

Understanding how proteins assemble into multicomponent complexes and how subunits within the complex interact remains a cornerstone of structural biology. It has been previously demonstrated that surface-induced dissociation is a useful tool for studying protein complex substructure and connectivity. When SID studies are performed on proteins prepared in AmAc in positive mode ionization, the larger substructural fragments are often lost with higher intensity monomer, dimer, etc. often being observed instead. Therefore, SID studies are routinely performed under charge reducing conditions, typically through the addition of TEAA, to obtain the most native-like fragmentation and hence substructural information however the addition of TEAA can often lead to increased adducting and peak broadening. Lower charge states can, however, be generated from negative mode ESI without the need for additives. We demonstrated here that those lower charge state anions fragment in a manner consistent with the native structure and can be used to obtain substructural information for protein complexes. For the human $20 \mathrm{~S}$ proteosome, preparing the sample in AmAc and analyzing in negative mode allowed more complete characterization and higher mass accuracy of the proteosome, in particular the $7 \alpha-$ ring than when the sample was prepared in AmAc+TEAA and analyzed in positive mode. However, for in-depth proteoform characterization, analyzing the highest charge states generated (in AmAc in positive mode) was advantageous. Therefore, for future studies it may be advantageous to prepare samples in AmAc and perform structural SID studies in negative mode and proteoform characterization in positive mode, all from the same sample solution. The results presented here demonstrate that nMS and SID can be used for structural characterization at multiple levels from stoichiometry and subunit connectivity to identification of posttranslational modifications. The ability to do this in a single experiment enables rapid and detailed structural characterization to be performed on low sample amounts and is applicable to even heterogenous samples. 


\section{Supporting information}

Additional figures can be found online in the electronic supporting information.

\section{Author information}

Corresponding author:

*E-mail: wysocki.11@osu.edu

Z.L.V current address Analytical Research \& Development Mass Spectrometry, Merck \& Co., Inc., 2000 Galloping Hill Road Kenilworth, NJ 07033, USA

\section{Acknowledgements}

The authors thank Melody Holmquist (OSU), Mark Foster (OSU) and Paul Gollnick (SUNY Buffalo) for providing TRAP (NIH R01 GM120923) and Gili Ben-Nisan (Weizmann Institute) and Michal Sharon (Weizmann Institute) for providing the human proteasome sample. The authors gratefully acknowledge funding from the National Institutes of Health awarded to V.H.W (SID of positive and negative protein complexes in this work was supported by NIH R01GM113658 and modification of the QE UHMR was made possible through NIH P41GM128577).

\section{References}

(1) Alberts, B. The Cell as a Collection of Protein Machines: Preparing the Next Generation of Molecular Biologists. Cell 1998, 92 (3), 291-294. https://doi.org/10.1016/S00928674(00)80922-8.

(2) Petsko, G.; Ringe, D. Protein Structure and Function; Primers in Biology; Oxford University Press: Oxford, New York, 2008.

(3) Stefani, M.; Dobson, C. M. Protein Aggregation and Aggregate Toxicity: New Insights into Protein Folding, Misfolding Diseases and Biological Evolution. J. Mol. Med. 2003, 81 (11), 678-699. https://doi.org/10.1007/s00109-003-0464-5.

(4) Minor, D. L. The Neurobiologist's Guide to Structural Biology: A Primer on Why Macromolecular Structure Matters and How to Evaluate Structural Data. Neuron 2007, 54 (4), 511-533. https://doi.org/10.1016/j.neuron.2007.04.026.

(5) Ward, A. B.; Sali, A.; Wilson, I. A. Integrative Structural Biology. Science 2013, 339 (6122), 913-915. https://doi.org/10.1126/science.1228565.

(6) Murata, K.; Wolf, M. Cryo-Electron Microscopy for Structural Analysis of Dynamic Biological Macromolecules. Biochim. Biophys. Acta BBA - Gen. Subj. 2018, 1862 (2), 324-334. https://doi.org/10.1016/j.bbagen.2017.07.020. 
(7) Yamashita, M.; Fenn, J. B. Electrospray lon Source. Another Variation on the Free-Jet Theme. J. Phys. Chem. 1984, 88 (20), 4451-4459. https://doi.org/10.1021/j150664a002.

(8) Fenn, J. B.; Mann, M.; Meng, C. K.; Wong, S. F.; Whitehouse, C. M. Electrospray Ionization for Mass Spectrometry of Large Biomolecules. Science 1989, 246 (4926), 6471. https://doi.org/10.1126/science.2675315.

(9) Ben-Nissan, G.; Sharon, M. The Application of Ion-Mobility Mass Spectrometry for Structure/Function Investigation of Protein Complexes. Curr. Opin. Chem. Biol. 2018, 42, 25-33. https://doi.org/10.1016/j.cbpa.2017.10.026.

(10) Hilton, G. R.; Benesch, J. L. P. Two Decades of Studying Non-Covalent Biomolecular Assemblies by Means of Electrospray Ionization Mass Spectrometry. J. R. Soc. Interface 2012, 9 (70), 801-816. https://doi.org/10.1098/rsif.2011.0823.

(11) Liko, I.; Allison, T. M.; Hopper, J. T.; Robinson, C. V. Mass Spectrometry Guided Structural Biology. Curr. Opin. Struct. Biol. 2016, 40, 136-144. https://doi.org/10.1016/j.sbi.2016.09.008.

(12) Benesch, J. L.; Ruotolo, B. T. Mass Spectrometry: Come of Age for Structural and Dynamical Biology. Curr. Opin. Struct. Biol. 2011, 21 (5), 641-649. https://doi.org/10.1016/j.sbi.2011.08.002.

(13) Eschweiler, J. D.; Kerr, R.; Rabuck-Gibbons, J.; Ruotolo, B. T. Sizing Up Protein-Ligand Complexes: The Rise of Structural Mass Spectrometry Approaches in the Pharmaceutical Sciences. Annu. Rev. Anal. Chem. 2017, 10 (1), 25-44. https://doi.org/10.1146/annurevanchem-061516-045414.

(14) Stiving, A. Q.; VanAernum, Z. L.; Busch, F.; Harvey, S. R.; Sarni, S. H.; Wysocki, V. H. Surface-Induced Dissociation: An Effective Method for Characterization of Protein Quaternary Structure. Anal. Chem. 2019, 91 (1), 190-209. https://doi.org/10.1021/acs.analchem.8b05071.

(15) Konijnenberg, A.; Butterer, A.; Sobott, F. Native lon Mobility-Mass Spectrometry and Related Methods in Structural Biology. Biochim. Biophys. Acta BBA - Proteins Proteomics 2013, 1834 (6), 1239-1256. https://doi.org/10.1016/j.bbapap.2012.11.013.

(16) Benesch, J. L. P.; Aquilina, J. A.; Ruotolo, B. T.; Sobott, F.; Robinson, C. V. Tandem Mass Spectrometry Reveals the Quaternary Organization of Macromolecular Assemblies. Chem. Biol. 2006, 13 (6), 597-605. https://doi.org/10.1016/j.chembiol.2006.04.006.

(17) Leney, A. C.; Heck, A. J. Native Mass Spectrometry: What Is in the Name? J Am Soc Mass Spectrom 2017, 28 (1), 5-13.

(18) Konermann, L.; Douglas, D. J. Unfolding of Proteins Monitored by Electrospray Ionization Mass Spectrometry: A Comparison of Positive and Negative lon Modes. J. Am. Soc. Mass Spectrom. 1998, 9 (12), 1248-1254. https://doi.org/10.1016/S1044-0305(98)001032.

(19) Heck, A. J. R.; Heuvel, R. H. H. van den. Investigation of Intact Protein Complexes by Mass Spectrometry. Mass Spectrom. Rev. 2004, 23 (5), 368-389. https://doi.org/10.1002/mas.10081.

(20) Allen, S. J.; Schwartz, A. M.; Bush, M. F. Effects of Polarity on the Structures and Charge States of Native-Like Proteins and Protein Complexes in the Gas Phase. Anal. Chem. 2013, 85 (24), 12055-12061. https://doi.org/10.1021/ac403139d.

(21) Liko, I.; Hopper, J. T. S.; Allison, T. M.; Benesch, J. L. P.; Robinson, C. V. Negative lons Enhance Survival of Membrane Protein Complexes. J. Am. Soc. Mass Spectrom. 2016, 27 (6), 1099-1104. https://doi.org/10.1007/s13361-016-1381-5.

(22) Konermann, L.; Ahadi, E.; Rodriguez, A. D.; Vahidi, S. Unraveling the Mechanism of Electrospray lonization. Anal. Chem. 2013, 85 (1), 2-9. https://doi.org/10.1021/ac302789c. 
(23) Kebarle, P.; Verkerk, U. H. Electrospray: From Ions in Solution to lons in the Gas Phase, What We Know Now. Mass Spectrom. Rev. 2009, 28 (6), 898-917.

https://doi.org/10.1002/mas.20247.

(24) Fernandez de la Mora, J. Electrospray Ionization of Large Multiply Charged Species

Proceeds via Dole's Charged Residue Mechanism. Anal. Chim. Acta 2000, 406 (1), 93104. https://doi.org/10.1016/S0003-2670(99)00601-7.

(25) Felitsyn, N.; Peschke, M.; Kebarle, P. Origin and Number of Charges Observed on Multiply-Protonated Native Proteins Produced by ESI. Int. J. Mass Spectrom. 2002, 219 (1), 39-62. https://doi.org/10.1016/S1387-3806(02)00588-2.

(26) Hall, Z.; Politis, A.; Bush, M. F.; Smith, L. J.; Robinson, C. V. Charge-State Dependent Compaction and Dissociation of Protein Complexes: Insights from lon Mobility and Molecular Dynamics. J. Am. Chem. Soc. 2012, 134 (7), 3429-3438. https://doi.org/10.1021/ja2096859.

(27) Dyachenko, A.; Gruber, R.; Shimon, L.; Horovitz, A.; Sharon, M. Allosteric Mechanisms Can Be Distinguished Using Structural Mass Spectrometry. Proc. Natl. Acad. Sci. 2013, 110 (18), 7235-7239. https://doi.org/10.1073/pnas.1302395110.

(28) Pacholarz, K. J.; Barran, P. E. Use of a Charge Reducing Agent to Enable Intact Mass Analysis of Cysteine-Linked Antibody-Drug-Conjugates by Native Mass Spectrometry. EuPA Open Proteomics 2016, 11, 23-27. https://doi.org/10.1016/j.euprot.2016.02.004.

(29) Stiving, A. Q.; Jones, B. J.; Ujma, J.; Giles, K.; Wysocki, V. H. Collision Cross Sections of Charge-Reduced Proteins and Protein Complexes: A Database for Collision Cross Section Calibration. Anal. Chem. 2020, 92 (6), 4475-4483. https://doi.org/10.1021/acs.analchem.9b05519.

(30) Schwartz, B. L.; Bruce, J. E.; Anderson, G. A.; Hofstadler, S. A.; Rockwood, A. L.; Smith, R. D.; Chilkoti, A.; Stayton, P. S. Dissociation of Tetrameric lons of Noncovalent Streptavidin Complexes Formed by Electrospray Ionization. J. Am. Soc. Mass Spectrom. 1995, 6 (6), 459-465. https://doi.org/10.1016/1044-0305(95)00191-F.

(31) Sinelnikov, I.; Kitova, E. N.; Klassen, J. S. Influence of Coulombic Repulsion on the Dissociation Pathways and Energetics of Multiprotein Complexes in the Gas Phase. J. Am. Soc. Mass Spectrom. 2007, 18 (4), 617-631. https://doi.org/10.1016/j.jasms.2006.11.006.

(32) Sciuto, S. V.; Liu, J.; Konermann, L. An Electrostatic Charge Partitioning Model for the Dissociation of Protein Complexes in the Gas Phase. J. Am. Soc. Mass Spectrom. 2011, 22 (10), 1679. https://doi.org/10.1007/s13361-011-0205-x.

(33) Popa, V.; Trecroce, D. A.; McAllister, R. G.; Konermann, L. Collision-Induced Dissociation of Electrosprayed Protein Complexes: An All-Atom Molecular Dynamics Model with Mobile Protons. J. Phys. Chem. B 2016, 120 (23), 5114-5124. https://doi.org/10.1021/acs.jpcb.6b03035.

(34) Zhou, M.; Wysocki, V. H. Surface Induced Dissociation: Dissecting Noncovalent Protein Complexes in the Gas Phase. Acc. Chem. Res. 2014, 47 (4), 1010-1018. https://doi.org/10.1021/ar400223t.

(35) Quintyn, R. S.; Yan, J.; Wysocki, V. H. Surface-Induced Dissociation of Homotetramers with D2 Symmetry Yields Their Assembly Pathways and Characterizes the Effect of Ligand Binding. Chem. Biol. 2015, 22 (5), 583-592.

https://doi.org/10.1016/j.chembiol.2015.03.019.

(36) Harvey, S. R.; Seffernick, J. T.; Quintyn, R. S.; Song, Y.; Ju, Y.; Yan, J.; Sahasrabuddhe, A. N.; Norris, A.; Zhou, M.; Behrman, E. J.; Lindert, S.; Wysocki, V. H. Relative Interfacial Cleavage Energetics of Protein Complexes Revealed by Surface Collisions. Proc. Natl. Acad. Sci. 2019, 116 (17), 8143-8148. https://doi.org/10.1073/pnas.1817632116. 
(37) Zhou, M.; Dagan, S.; Wysocki, V. H. Impact of Charge State on Gas-Phase Behaviors of Noncovalent Protein Complexes in Collision Induced Dissociation and Surface Induced Dissociation. Analyst 2013, 138 (5), 1353-1362. https://doi.org/10.1039/C2AN36525A.

(38) Quintyn, R. S.; Zhou, M.; Yan, J.; Wysocki, V. H. Surface-Induced Dissociation Mass Spectra as a Tool for Distinguishing Different Structural Forms of Gas-Phase Multimeric Protein Complexes. Anal. Chem. 2015, 87 (23), 11879-11886. https://doi.org/10.1021/acs.analchem.5b03441.

(39) McElroy, C.; Manfredo, A.; Wendt, A.; Gollnick, P.; Foster, M. TROSY-NMR Studies of the $91 \mathrm{kDa}$ TRAP Protein Reveal Allosteric Control of a Gene Regulatory Protein by Ligand-Altered Flexibility. J. Mol. Biol. 2002, 323 (3), 463-473.

https://doi.org/10.1016/S0022-2836(02)00940-3.

(40) Holmquist, M. L.; Ihms, E. C.; Gollnick, P.; Wysocki, V. H.; Foster, M. P. Population Distributions from Native Mass Spectrometry Titrations Reveal Nearest-Neighbor Cooperativity in the Ring-Shaped Oligomeric Protein TRAP. Biochemistry 2020, 59 (27), 2518-2527. https://doi.org/10.1021/acs.biochem.0c00352.

(41) Ruschak, A. M.; Religa, T. L.; Breuer, S.; Witt, S.; Kay, L. E. The Proteasome Antechamber Maintains Substrates in an Unfolded State. Nature 2010, 467 (7317), 868871. https://doi.org/10.1038/nature09444.

(42) Vimer, S.; Ben-Nissan, G.; Morgenstern, D.; Kumar-Deshmukh, F.; Polkinghorn, C.; Quintyn, R. S.; Vasil'ev, Y. V.; Beckman, J. S.; Elad, N.; Wysocki, V. H.; Sharon, M. Comparative Structural Analysis of 20S Proteasome Ortholog Protein Complexes by Native Mass Spectrometry. ACS Cent. Sci. 2020, 6 (4), 573-588. https://doi.org/10.1021/acscentsci.0c00080.

(43) Busch, F.; VanAernum, Z. L.; Ju, Y.; Yan, J.; Gilbert, J. D.; Quintyn, R. S.; Bern, M.; Wysocki, V. H. Localization of Protein Complex Bound Ligands by Surface-Induced Dissociation High-Resolution Mass Spectrometry. Anal. Chem. 2018, 90 (21), 1279612801. https://doi.org/10.1021/acs.analchem.8b03263.

(44) VanAernum, Z. L.; Gilbert, J. D.; Belov, M. E.; Makarov, A. A.; Horning, S. R.; Wysocki, V. $\mathrm{H}$. Surface-Induced Dissociation of Noncovalent Protein Complexes in an Extended Mass Range Orbitrap Mass Spectrometer. Anal. Chem. 2019, 91 (5), 3611-3618. https://doi.org/10.1021/acs.analchem.8b05605.

(45) Harvey, S. R.; VanAernum, Z. L.; Kostelic, M. M.; Marty, M. T.; Wysocki, V. H. Probing the Structure of Nanodiscs Using Surface-Induced Dissociation Mass Spectrometry. Chem. Commun. 2020. https://doi.org/10.1039/D0CC05531J.

(46) Pagel, K.; Hyung, S.-J.; Ruotolo, B. T.; Robinson, C. V. Alternate Dissociation Pathways Identified in Charge-Reduced Protein Complex lons. Anal. Chem. 2010, 82 (12), 53635372. https://doi.org/10.1021/ac101121r.

(47) Shrive, A. K.; Gheetham, G. M. T.; Holden, D.; Myles, D. A. A.; Turnell, W. G.; Volanakis, J. E.; Pepys, M. B.; Bloomer, A. C.; Greenhough, T. J. Three Dimensional Structure of Human C-Reactive Protein. Nat. Struct. Biol. 1996, 3 (4), 346-354. https://doi.org/10.1038/nsb0496-346.

(48) Harvey, S. R.; Yan, J.; Brown, J. M.; Hoyes, E.; Wysocki, V. H. Extended Gas-Phase Trapping Followed by Surface-Induced Dissociation of Noncovalent Protein Complexes. Anal. Chem. 2016, 88 (2), 1218-1221. https://doi.org/10.1021/acs.analchem.5b03479.

(49) Marty, M. T.; Baldwin, A. J.; Marklund, E. G.; Hochberg, G. K. A.; Benesch, J. L. P.; Robinson, C. V. Bayesian Deconvolution of Mass and Ion Mobility Spectra: From Binary Interactions to Polydisperse Ensembles. Anal. Chem. 2015, 87 (8), 4370-4376. https://doi.org/10.1021/acs.analchem.5b00140.

(50) Schrader, J.; Henneberg, F.; Mata, R. A.; Tittmann, K.; Schneider, T. R.; Stark, H.; Bourenkov, G.; Chari, A. The Inhibition Mechanism of Human $20 \mathrm{~S}$ Proteasomes Enables 
Next-Generation Inhibitor Design. Science 2016, 353 (6299), 594-598.

https://doi.org/10.1126/science.aaf8993.

(51) Krissinel, E.; Henrick, K. Inference of Macromolecular Assemblies from Crystalline State. J. Mol. Biol. 2007, 372 (3), 774-797. https://doi.org/10.1016/j.jmb.2007.05.022. 